\title{
Assessment of core and support functions of case- based surveillance of meningitis in hospitals in Khartoum State in 2015
}

I.A.A. Baghdadi ${ }^{\top}$

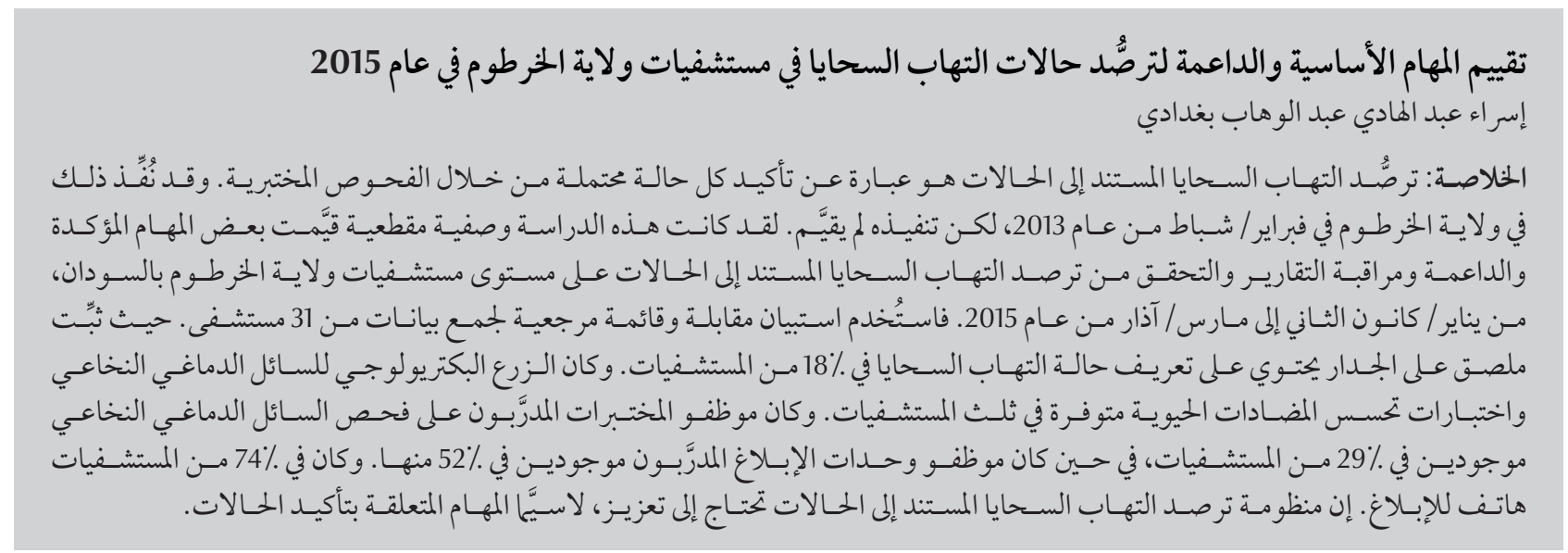

ABSTRACT Case-based surveillance of meningitis was implemented in Khartoum State in February 2013 but its implementation has not been evaluated. This study assessed some core (case confirmation) and support (case reporting and investigation resources) functions of case-based surveillance of meningitis at hospital level in Khartoum State, Sudan, from January to March 2015. An interview questionnaire and checklist were used to collect data from 31 hospitals. A poster containing the case definition of meningitis was fixed to the wall in 18\% of the hospitals. Bacteriological culture of cerebrospinal fluid (CSF) and antibiotic sensitivity testing of the cultured bacteria were available in one-third of the hospitals. Laboratory personnel trained in CSF examination were present in $29 \%$ of the hospitals, while trained reporting unit personnel were present in 52\%. A telephone for reporting was present in $74 \%$ of the hospitals. The system for casebased surveillance of meningitis needs to be strengthened, particularly the functions related to case confirmation.

Évaluation des fonctions essentielles et des fonctions d'appui du système de surveillance des cas de méningite dans les hôpitaux de l'État de Khartoum en 2015

RÉSUMÉ La surveillance des cas de méningite a été mise en place dans l'État de Khartoum en février 2013, mais sa mise en œuvre n'a pas fait l'objet d'une évaluation. La présente étude avait pour objectif d'évaluer les fonctions essentielles (confirmation des cas) et d'appui (notification des cas et ressources pour l'investigation) du système de surveillance des cas de méningite à l'échelle des hôpitaux dans l'État de Khartoum, au Soudan, de janvier à mars 2015. Un questionnaire d'entrevue et une liste de contrôle ont été utilisés pour collecter les données sur 31 hôpitaux. Une affiche présentant la définition d'un cas de méningite a été accrochée sur les murs de 18 \% des hôpitaux. L'analyse bactériologique du liquide céphalorachidien (LCR) et un test de sensibilité aux antibiotiques de la bactérie mise en culture étaient disponibles dans un tiers des hôpitaux. 29 \% des hôpitaux disposaient de personnel de laboratoire formé à l'analyse du LCR, et 52 \% d'équipes chargées de la notification des cas. Une ligne téléphonique destinée aux signalements était présente dans 74 \% des hôpitaux. Le système de surveillance des cas de méningite doit être renforcé, en particulier les fonctions liées à la confirmation des cas. 


\section{Introduction}

Epidemic meningitis has been observed in the African meningitis belt [an area that extends from Senegal to Ethiopia) for over a century (1)]. Initially, surveillance was limited to counting suspected cases and deaths. However, progress in microbiological techniques led to etiological surveillance and observation of strain epidemiology, and thresholds were set for the incidence of suspected cases to guide vaccine response during epidemics The prime objective of surveillance over the next few years will be to evaluate the impact of meningococcal A conjugate vaccine and pneumococcal conjugate vaccine in several if not all countries in the meningitis belt (2)

Case-based surveillance of meningitis is the confirmation of every probable case through laboratory testing, to determine the epidemiological and microbiological profile of each case (3), and it was implemented in Khartoum State in February 2013 (4). Pneumococcal conjugate vaccine 13 was included as part of routine vaccination in August 2013 (5). Mass vaccination campaigns with meningococcal A conjugate vaccine (MenAfriVac) targeting people aged 1-29 years in Khartoum State was conducted in 7-18 October 2012, with $95 \%$ coverage (6). The number of suspected cases of meningitis in Khartoum State has fluctuated annually since 2008 (Figure 1), and the marked reduction in 2014 may in part resulted from changing the case definition to include fewer criteria (Annual reports of meningitis from 2008 to 2014; weekly reports of meningitis for 2015. Department of Surveillance, Directorate of Preparedness and Response of Epidemics, Federal Ministry of Health, Sudan). Monitoring and evaluation are crucial for strengthening surveillance and response systems, and they should be an integral part of these systems (7).

The first objective of this study was to assess the core functions of the surveillance system with regard to availability of posters fixed to the wall in outpatient clinics with the case definition of meningitis, laboratory diagnosis, and feedback from the localities to the hospitals about the received reports. The second objective was to assess the support functions of the surveillance system with regard to availability of standard operating procedures and forms for cerebrospinal fluid (CSF) examination, case investigation and reporting; availability of telephones for reporting; and whether the personnel in the laboratory and the reporting units in the hospitals received training in case-based surveillance of meningitis.

\section{Methods}

This study was carried out from January to March 2015. The study population consisted of 15 general, 10 rural and 6 paediatric government hospitals in Khartoum State. An interview questionnaire was used to collect data from the laboratories where the chief personnel or someone assigned by them was interviewed, and from the reporting units.
The public health officers who were away from the hospital at the time of the visit were interviewed by telephone. The questionnaire was adapted from the WHO generic questionnaire (8). The first part concerned laboratory-related functions: availability of culture and sensitivity tests; availability of request forms and standard operating procedures for CSF examination; and training of laboratory personnel for CSF examination. The second part concerned functions related to the reporting unit: availability of a telephone for reporting; availability of standard operating procedures and forms for case reporting and investigation; and training of personnel and whether they received feedback from the localities about the received reports. We checked whether there was a poster containing the case definition of meningitis fixed to the wall in the outpatient clinics. Two hospitals were contacted through telephone only and were not visited (Abudelig and Omkati Hospitals). The data were analysed using SPSS, version 20 where only descriptive analysis was conducted (only counting

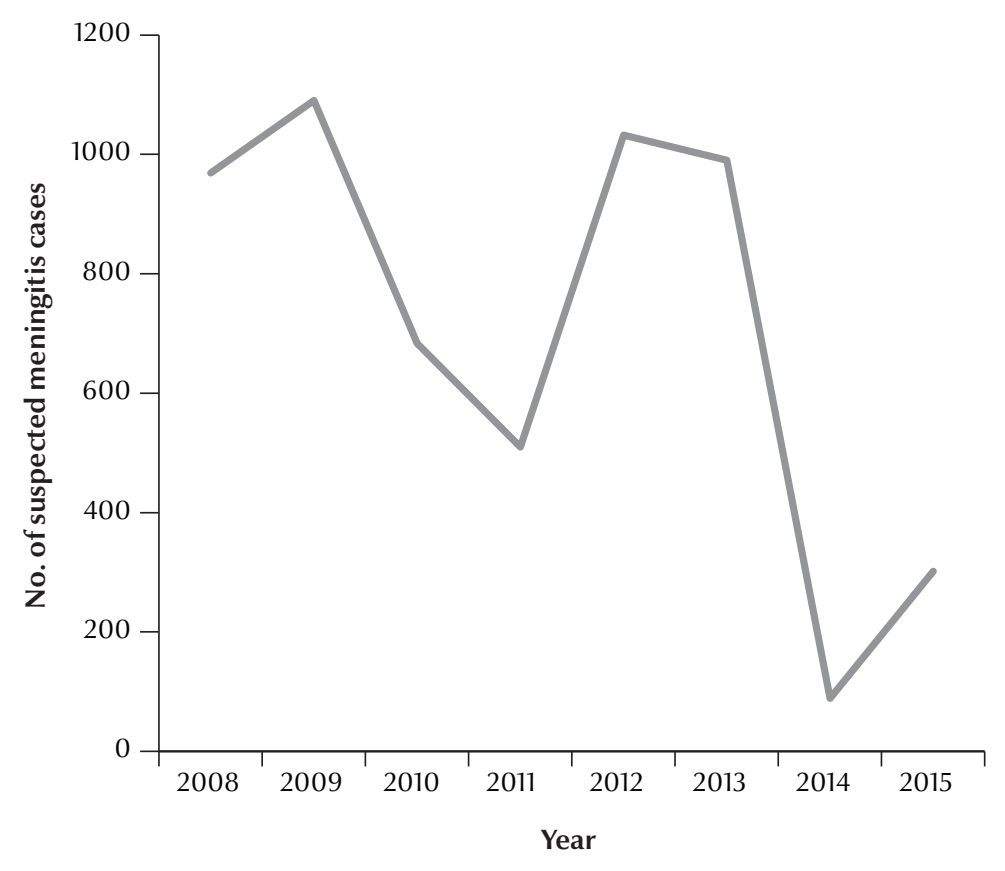

Figure 1 Number of suspected cases of meningitis in Khartoum state, 2008September 2015 
availability of the functions in the different hospital categories.)

\section{Results}

Table 1 shows the availability of functions related to case confirmation in case-based surveillance of meningitis in hospitals in Khartoum State in 2015. These functions were not present in rural hospitals.

A poster fixed to the wall, containing the case definition of meningitis, was present in 5 of the 28 hospitals that received emergency cases; in 1 , it was a poster of list $B$ diseases (an old poster in which meningitis was classified with the diseases that are reported on a weekly basis), and in the other 4, it showed guidelines for dealing with epidemic meningitis. A poster was also present in another 2 hospitals but 1 was on the floor while the other was on the window.

For suspected cases of meningitis in the 20 hospitals that had no culture and sensitivity tests: 12 hospitals refer the cases to another hospital; 3 refer CSF samples to another hospital; 2 refer the CSF samples to the National Public Health Laboratory; 1 refers the samples to Khartoum State Public Health Laboratory; 1 performs only CSF sugar, protein and white blood cell count; and 1 refers the samples to another hospital for culture and sensitivity tests if the initial results for sugar, protein and white blood cell count suggest meningitis.

Table 2 shows the functions related to case reporting and investigation in case-based surveillance of meningitis in hospitals in Khartoum State in 2015.

\section{Discussion}

The functions related to case confirmation were absent in the rural hospitals but they were available to varying degrees in general and paediatric hospitals. The functions related to case reporting and investigation were to some extent comparable in rural, general and paediatric hospitals.

The request form for CSF examination is available in only one hospital which is a sentinel site for the rotavirus and invasive bacterial diseases surveillance network, and it is provided by the network.

Culture and sensitivity tests were available in 11 hospitals (35\%), which was better than in Uganda (9), where only $21 \%$ of the health facilities can confirm meningococcal meningitis, and in India (10), where CSF examination is only available in referral laboratories. A previous study in Khartoum State (11) reported that $<25 \%$ of the facilities were able to collect CSF specimens, but it was not determined whether they could perform culture and sensitivity tests. Of the 20 hospitals that had no culture and sensitivity tests, 12 refer the patients to other hospitals, which is consistent with India, where most of the hospitals refer the patients rather than the specimens (10). The culture and sensitivity tests

\begin{tabular}{|c|c|c|c|}
\hline Function & $\begin{array}{l}\text { Rural hospitals } \\
\qquad(\mathbf{1 0})\end{array}$ & $\begin{array}{l}\text { General hospitals } \\
\text { (15) }\end{array}$ & $\begin{array}{l}\text { Paediatric hospitals } \\
\text { (6) }\end{array}$ \\
\hline Availability of CSF test request form & 0 & 0 & 1 \\
\hline Availability of culture and sensitivity tests & 0 & 8 & 3 \\
\hline $\begin{array}{l}\text { Availability of standard operating procedures for CSF } \\
\text { examination }\end{array}$ & 0 & 3 & 3 \\
\hline $\begin{array}{l}\text { Availability of laboratory personnel trained in CSF } \\
\text { examination }\end{array}$ & 0 & 4 & 5 \\
\hline
\end{tabular}

CSF = cerebrospinal fluid.

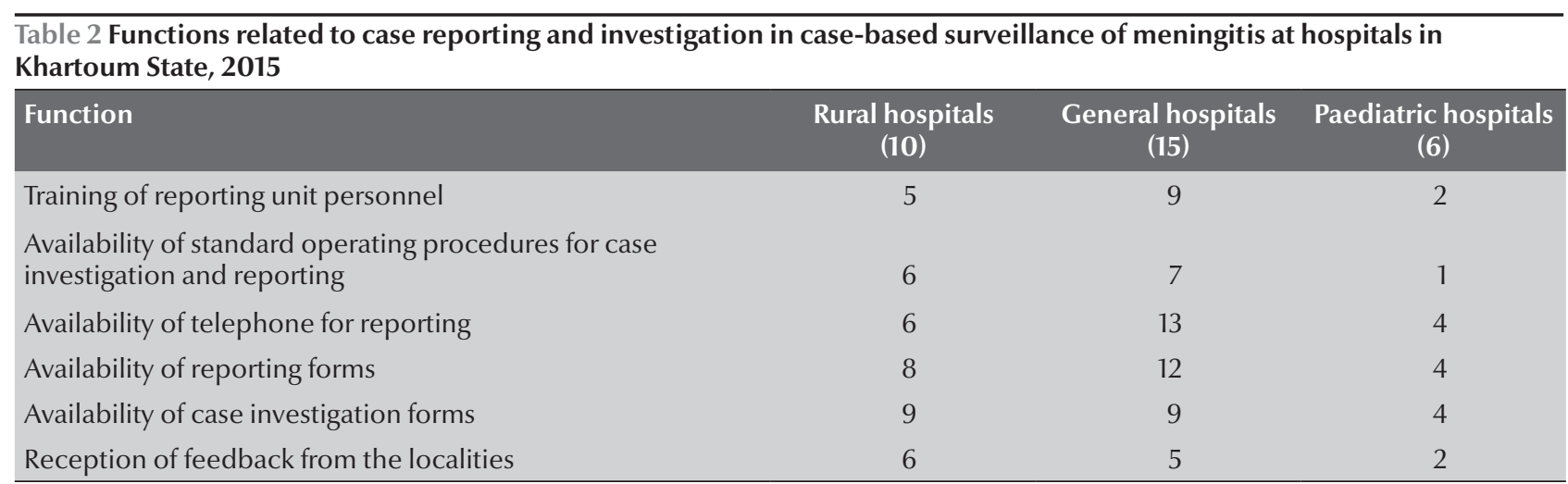


were not available in any of the rural hospitals, which could be explained by the lack of stable electricity supply and qualified laboratory personnel.

The standard operating procedures for CSF examination were present in 6 hospitals (19\%), and only 3 of these were part of the 11 that perform culture and sensitivity tests. In contrast, the standard operating procedures for case reporting and investigation were present in 14 hospitals (45\%). The discrepancy between the laboratory functions and the functions related to case reporting and investigation might be partly related to the different administration of the two departments and lack of coordination between them.

The reporting units personnel were trained in 16 hospitals (52\%). This was similar to a study in Ethiopia in which $52 \%$ of the health facilities had at least one person trained in surveillance of acute flaccid paralysis (12). In contrast, the situation was better in Uganda, where they reported that $62 \%$ of the personnel in health facilities received training in epidemiology (9).

Twenty-three of the 31 hospitals (74\%) had a telephone for case reporting, which is lower than was previously found in Khartoum State (89.3\%) (11), but better than found in Uganda (27\%) (9).

Case investigation forms were present in $22(71 \%)$ of the hospitals and case reporting forms in 24 (77\%). This is inferior to the $100 \%$ that was reported in Khartoum State (11) and Ethiopia (12), but better than the $35 \%$ reported in Uganda (9). The lack of the forms in the hospitals was mainly caused by lack of follow-up of supplies.

Only 13 hospitals (42\%) received feedback from the localities about the reports received from the hospitals, however, this was better than the $15 \%$ in Uganda, India and Ethiopia $(9,10,12)$. Again, the problem of receiving feedback was the result of poor follow-up because 1 feedback report is prepared by the locality for all the hospitals, but it is received in some but not in others in the same locality. In the previous study in Khartoum State, it was reported that all the localities and health areas produced a regular feedback report to the lower level (hospitals and health centres) but they did not document whether it was received at the hospitals (11).
Our study had the following limitations: (1) assessment was limited to hospitals only; (2) 2 hospitals were only contacted through telephone and were not visited; (3) public health officers who were outside the hospital at the time of the visit were interviewed by telephone; and (4) not all the core and support functions were addressed.

To conclude, case-based surveillance of meningitis in Khartoum State was found to be weak with regard to functions related to case confirmation. Such functions were not available in any of the rural hospitals and in only about half of the general and paediatric hospitals. The functions related to case reporting were better, especially availability of telephones and case reporting and investigation forms The situation can be improved by training laboratory and reporting unit personnel in all the hospitals, provision of the required supplies, and formulation and distribution of a protocol outlining how to deal with CSF samples in case the tests are not available in the hospital.

Funding: None.

Competing interests: None declared.

\section{References}

1. Standard operating procedures for enhanced meningitis surveillance in Africa: African 'Meningitis Belt'. Brazzaville: World Health Organization Regional Office for Africa; 2011 (http:// apps.who.int/iris/handle/10665/1906, accessed 4 May 2015).

2. Mueller JE. Conjugate vaccine introduction in the African meningitis belt: meeting surveillance objectives. Trop Med Int Health. 2013 Jan;18(1):58-64. PMID:23121399

3. The manual of case based surveillance of meningitis in Sudan. Khartoum: Department of Epidemiology and Zoonotic Diseases, General Directorate of Primary Health Care, Federal Ministry of Health; 2013.

4. The annual report of meningitis. Khartoum: Department of Surveillance, Directorate of Preparedness and Response of Epidemics, Federal Ministry of Health; 2013.

5. The annual report of the expanded programme on immunization. Khartoum: Federal Ministry of Health; 2013.

6. The coverage report of the MenAfrivac campaign, Expanded Programme of Immunization, Khartoum: Federal Ministry of Health.

7. Overview of the WHO framework for monitoring and evaluating surveillance and response systems for communicable diseases. Wkly Epidemiol Rec. 2004 Sep 3;79(36):321-6.
8. Protocol for the assessment of national communicable disease surveillance and response systems. WHO/CDS/CSR/ ISR/2001.2. Annex 12. Generic questionnaires (http://www. who.int/csr/resources/publications/surveillance/whocdscsrisr20012a.pdf?ua=1, accessed 26 April 2016)

9. Assessment of infectious disease surveillance - Uganda, 2000. MMWR Morb Mortal Wkly Rep. 2000 Aug 4;49(30):687-91.

10. Phalkey RK, Shukla S, Shardul S, Ashtekar N, Valsa S, Awate $P$, et al. Assessment of the core and support functions of the Integrated Disease Surveillance system in Maharashtra, India. BMC Public Health. 2013 Jun 13;13(1):575. PMID:23764137

11. Sahal N, Reintjes R, Eltayeb E, Aro AR. Assessment of core activities and supportive functions for the communicable diseases surveillance systemin Khartoum state, Sudan, 2005-2007. East Mediterr Health J. 2010;16(12):1204-10.

12. Assessment of the national communicable disease surveillance and response system, Ethiopia. Wkly Epidemiol Rec. 2001 Jan 12;76(2):9-16. 